\title{
Parametrização hierárquica de superfícies poligonais construída com triangulação de Delaunay restrita
}

\author{
Pablo V. F. Telles *, Romildo Silva ${ }^{\dagger}$, Esdras Medeiros ${ }^{\dagger}$ e Sinésio Pesco * \\ *Departamento de Matemática - Pontifícia Universidade Católica do Rio de Janeiro - PUC-Rio \\ Rio de Janeiro, RJ, Brasil \\ †Departamento de Matemática - Universidade Federal do Ceará - UFC \\ Fortaleza, CE, Brasil \\ Email: pablotelles21@gmail.com
}

\begin{abstract}
The triangulated surfaces can be parametrized hierarchically allow a representation of the surface and of his parameter domain by levels of details and allow a better control of the parametrization. In this work we present a hierarchical structure for triangulated surfaces with the disk like topology and constructed with a restricted Delaunay triangulation. This construction is free of possible topological and foldover inconsistencies introduced in triangulation by the process of simplification. In addition, we use an hierarchical optimization process to reduce the distortions caused by parametrization.

Resumo-As superfícies triangularizadas podem ser parametrizadas hierarquicamente possibilitando uma representação da superfície, juntamente com o seu domínio paramétrico, por níveis de detalhes e, assim, permitindo um controle melhor da parametrização. Neste artigo apresentamos uma estrutura hierárquica para superfícies triangularizadas, com a topologia de um disco, construída com uma triangulação de Delaunay restrita. Essa construção é livre de possíveis inconsistências topológicas ou na orientação das faces introduzidas na triangulação pelo processo de simplificação. Além disso, utilizamos um processo de otimização na hierarquia para reduzir as distorções causadas pela parametrização.
\end{abstract}

\section{INTRODUÇÃO}

A parametrização de uma superfície tridimensional triangularizada $\mathcal{S}$ topologicamente equivalente ao disco é um mapeamento planar de $\mathcal{S}$. Esse mapeamento identifica bijetivamente os vértices desta superfície $\mathcal{S}$ com os vértices de uma triangulação planar $\mathcal{P}$ mantendo a relação de adjacências de $\mathcal{S}$ em $\mathcal{P}$. Assim, os triângulos de $\mathcal{S}$ possuem correspondência biunívoca com os triângulos de $\mathcal{P}$.

Em sentido mais amplo, uma superfície triangularizada compacta sem bordo não poderá ser parametrizada com um único domínio de parametrização planar. Desse modo, a nossa pesquisa busca desenvolver primordialmente um método robusto que parametrize esses tipos de superfícies utilizando uma estrutura de variedades diferenciáveis, construídas a partir de [1], reduzindo as distorções de ângulos e áreas introduzidas pelo processo de parametrização e construído hierarquicamente a partir da estrutura de multi-resolução proposta por [2].

Como resultado parcial, nesse artigo apresentamos um método hierárquico para parametrizações de superfícies homeomorfas a um disco construído a partir da simplificação da superfície original e realizamos uma otimização da energia de distorção introduzida por esse processo de parametrização.
A parametrização é utilizada em aplicações da computação gráfica como, por exemplo, texturização, compressão e remalhamento de superfícies triangularizadas. Diversos métodos, lineares ou não-lineares, têm sido desenvolvidos para a realização de parametrizações de malhas triangulares com o objetivo de equilibrar a redução das distorções introduzidas e manter uma eficiência computacional. Desta forma podemos obter de [3], [4], [5] e [6] uma visão geral de alguns dos principais métodos já desenvolvidos e das principais aplicações.

\section{ABordagem Proposta}

Inicialmente, foi introduzido por [7] uma estrutura hierárquica composta pela estratégia de simplificação para malhas, que são superfícies triangularizadas, baseada na operação edge collapse e pela sua representação inversa por meio da progressão da malha obtida pela operação vertex split. Essa estrutura permite que a superfície possa ser identificada com o nível de detalhes desejado. Nesse sentido, [8] apresenta uma estrutura hierárquica construída a partir da parametrização de superfícies com a topologia de um disco onde a etapa de simplificação é realizada com a operação half-edge collapse que é um caso particular da operação edge collapse.

A operação half-edge collapse pode introduzir inconsistências topológicas na superfície triangularizada $\mathcal{S}$ ao longo da etapa de simplificação, como apresentado por [9], quando as estrelas centradas nos vértices extremos da aresta escolhida tenha outros vértices de interseção além dos vértices opostos a esta aresta (figura 1b) ou inconsistências na orientação das faces da triangulação $\mathcal{P}$ (figura 1c) afetando a parametrização.

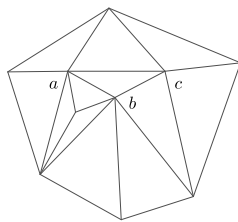

(a) Original

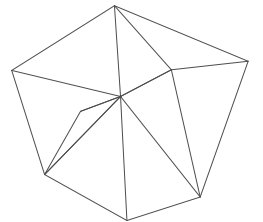

(b) Colapso: $a$ para $b$

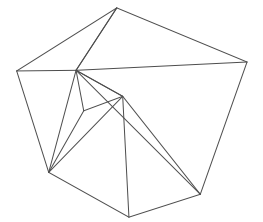

(c) Colapso: $c$ para $a$
Figura 1. Inconsistências

Com o objetivo de reduzir possíveis inconsistências topológicas ou de orientação das faces nas triangulações pla- 
nares associadas com as superfícies durante o processo de simplificação de $\mathcal{S}$, desde os níveis intermediários, substituímos a operação half-edge collapse pelas remoções de vértices e essas podem ser realizadas livremente. Para cada remoção obtemos uma nova triangulação para o buraco formado em $\mathcal{P}$ utilizando uma triangulação de Delaunay restrita ao bordo desse buraco. Ao longo do processo de simplificação obtemos diversas modificações locais na parametrização $\mathcal{P}$ e consequentemente na superfície $\mathcal{S}$.

O histórico das modificações realizadas pelo processo de simplificação fica registrado em uma estrutura hierárquica com $N$ níveis, onde cada nível $H_{i}$ desta estrutura é definido pela triangulação $\mathcal{P}_{i}$ e pelas informações que permitem reconstruir a triangulação $\mathcal{P}_{i+1}$, para $i \in\{0, \ldots, N-1\}$. Assim, teremos:

$$
\mathrm{MB}=\mathcal{S}_{0} \leftrightarrows \mathcal{S}_{1} \leftrightarrows \mathcal{S}_{2} \leftrightarrows \cdots \leftrightarrows \mathcal{S}_{N-1} \leftrightarrows \mathcal{S}_{N}=\mathcal{S}=\mathrm{MD}
$$

onde $\longleftarrow$ indica a simplificação da malha densa e $\longrightarrow$ indica o refinamento da malha base.

É desejável, portanto, que a parametrização preserve globalmente as propriedades geométricas ângulo e área. Entretanto, a manutenção total dessas propriedades geométricas, pela parametrização, só é possível nos casos em que a superfície $\mathcal{S}$ é desenvolvível [10], ou seja, em superfícies triangularizadas a soma das medidas dos ângulos incidentes em cada vértice interior da superfície é até $2 \pi$, o que não ocorre na maioria dos casos. Desse modo, utilizamos o processo apresentado em [11] que busca otimizar o funcional que mede a energia de distorção ao longo da hierarquia.

\section{CONSTRUÇÃO DA HIERARQUiA}

Partindo da superfície $\mathcal{S}$, a construção da hierarquia ocorre em duas etapas independentes. Na primeira etapa realizamos uma parametrização de $\mathcal{S}$ por meio de uma correspondência biunívoca $\phi: \mathcal{P} \rightarrow \mathcal{S}$ e, na segunda etapa, realizamos uma simplificação da triangulação $\mathcal{P}$ e, consequentemente, da superfície $\mathcal{S}$ registrando o histórico das modificações realizadas.

Neste contexto, a superfície $\mathcal{S}=(G, V)$ homeomorfa a um disco é composta pelo complexo simplicial $G$ que armazena as informações de conectividades entre vértices, arestas e faces e pelo conjunto $V=\left\{v_{1}, \ldots, v_{n}\right\} \subset \mathbb{R}^{3}$. Deste modo, $\mathcal{P}=(G, U)$ terá as mesmas conectividades e o conjunto $U=\left\{u_{1}, \ldots, u_{n}\right\} \subset \mathbb{R}^{2}$ tal que $\phi\left(u_{i}\right)=v_{i}$, para $i \in\{1, \ldots, n\}$. Além disso, é desejável que o mapa $\phi^{-1}$ associe triângulos de $\mathcal{S}$ com triângulos não degenerados de $\mathcal{P}$ mantendo a mesma orientação.

\section{A. Parametrização}

A construção da parametrização inicial $\phi$ é realizada com o mapa de combinação convexa introduzido por [12] e generalizado por [13] e [14], onde inicialmente os vértices do bordo de $\mathcal{S}$ são mapeados no bordo de $\mathcal{P}$ que é um polígono convexo planar e cada vértice interior de $\mathcal{S}$ será associado com um vértice interior de $\mathcal{P}$ por meio de uma combinação convexa dos demais vértices satisfazendo ao sistema de equações, $\sum_{j \in \mathcal{N}_{i}} w_{i j}\left(u_{i}-u_{j}\right)=0$, onde $\mathcal{N}_{i}=$ $\{j \mid(i, j)$ é uma aresta de $G\}$ define a vizinhança do $i$-ésimo vértice, para $i \in\{1, \ldots, n\}$, e $w_{i j}>0$ são pesos definidos para cada aresta $(i, j)$ de $G$.

Os pesos foram escolhidos como coordenadas do valor médio de acordo com [15] e assim,

$$
w_{i j}=\frac{\tan \left(\gamma_{i j} / 2\right)-\tan \left(\delta_{i j} / 2\right)}{\left\|v_{i}-v_{j}\right\|},
$$

para cada aresta $(i, j)$ de $G$ onde $\gamma_{i j}$ e $\delta_{i j}$ são os ângulos que possuem o vértice $v_{i}$ e que são internos aos triângulos de $\mathcal{S}$ que compartilham a aresta $(i, j)$. Esses pesos variam suavemente e garantem que o mapa de parametrização $\phi$ seja bijetivo e preserve a orientação, como mostrado por [15].

\section{B. Simplificação}

Após a etapa de parametrização o conjunto das arestas de $\mathcal{S}$ é agrupado em uma fila de prioridades de acordo com o comprimento. Este critério nos permite escolher a aresta com o menor tamanho e, nesta aresta, eliminar o vértice interior de $\mathcal{S}$ que é o centro da estrela com a menor média dos comprimentos das suas arestas. A eliminação do vértice também ocorrerá por correspondência em $\mathcal{P}$ destruindo a sua estrela e criando um buraco limitado pela curva poligonal que é o link dessa estrela removida.

A triangulação de Delaunay restrita proposta em [16] é utilizada para refazer a triangulação de cada buraco formado em $\mathcal{P}$ sendo o bordo deste buraco a restrição e podendo ser este bordo um polígono convexo ou não-convexo. Consequentemente, uma nova triangulação será refeita em $\mathcal{S}$ e procedemos repetindo estas eliminações até que a simplificação atinja um limite desejado. Essa triangulação de Delaunay construída no buraco formado em $\mathcal{P}$ é realizada com $\mathcal{O}(k \log (k))$, sendo $k$ a valência do vértice removido.

Durante a simplificação cada estrela removida na superfície $\mathcal{S}$ corresponde a uma estrela removida na triangulação $\mathcal{P}$. Com isso, podemos associar, na triangulação $\mathcal{P}$, o vértice que é o centro da estrela removida com um dos triângulos da nova triangulação refeita no buraco (figura 2). O triângulo associado ao vértice removido é exatamente aquele que contém a antiga posição geométrica deste vértice.

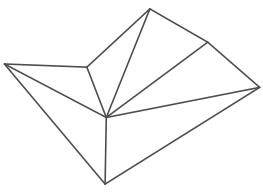

(a) Estrela original

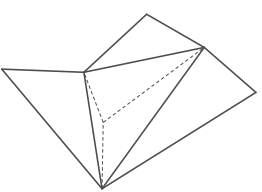

(b) Nova triangulação
Figura 2. Triangulação de Delaunay restrita

Assim, um histórico da simplificação é criado armazenando para cada remoção: $(i)$ as coordenadas baricêntricas do vértice removido em relação ao triângulo associado (relação indicada pelo pontilhamento na figura 2b); (ii) os índices dos vértices do triângulo associado; (iii) os índices dos vértices do link da estrela removida. Com estas informações podemos partir da superfície simplificada e realizar, no sentido inverso, um refinamento progressivo até recuperarmos a superfície $\mathcal{S}$ original. 
Inconsistências topológicas ou de orientação como a representada pela figura 1 podem ocorrer naturalmente na triangulação planar se a simplificação for baseada na operação de half-edge collapse o que requer um tratamento adicional na escolha do vértice a ser colapsado por esta operação. A nossa estrutura hierárquica evita essas inconsistências por utilizar uma nova triangulação para a estrela de cada vértice removido na triangulação planar e sendo esta uma triangulação de Delaunay local. Para cada remoção de vértice com valência $k$ são removidos $k$ triângulos e criados $k-2$ novos triângulos.

A figura 3 apresenta alguns níveis da hierarquia para o modelo Nefertiti onde as superfícies no nível $H_{0}$ tem 351 vértices e 564 faces, no nível $H_{1022}$ tem 1373 vértices e 2608 faces, no nível $H_{2044}$ tem 2395 vértices e 4652 faces, e por fim a superfície original com 4439 vértices e 8740 faces.

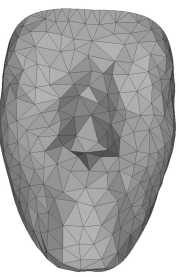

(a) $\mathcal{S}_{0}$

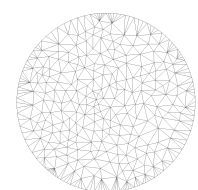

(e) $\mathcal{P}_{0}$

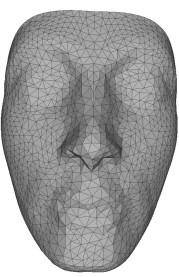

(b) $\mathcal{S}_{1022}$

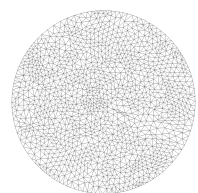

(f) $\mathcal{P}_{1022}$

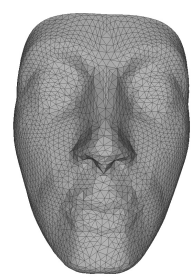

(c) $\mathcal{S}_{2044}$

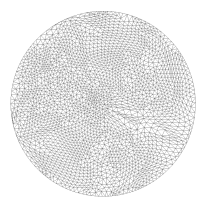

(g) $\mathcal{P}_{2044}$

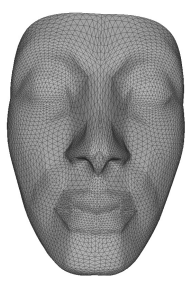

(d) $\mathcal{S}_{4088}$
Figura 3. Alguns níveis da hierarquia

\section{ENERGIA DE DISTORÇÃo}

Os métodos de parametrização desenvolvidos para superfícies poligonais quase sempre causam distorções no ângulo ou na área, isto é, os triângulos da superfície tridimensional sofrem deformações quando mapeados no plano.

Neste artigo utilizamos um funcional que combina a energia da área e do ângulo simultaneamente e é apresentado por [11]. Seja $\phi: \Omega \subset \mathbb{R}^{2} \rightarrow \mathcal{S}$ o mapa da parametrização de $\mathcal{S}$, de modo que $\phi^{-1}(\mathcal{S})=\mathcal{P}$. Para cada $\omega \in \mathcal{P}$ este funcional é definido por $E(\omega)=E_{\text {ângulo }}(w) \cdot E_{\text {area }}(w)^{\theta}$, onde $E_{\text {ângulo }} \mathrm{e}$ $E_{\text {área }}$ são funcionais que medem, respectivamente, a energia da distorção angular e da área, e $\theta \geqslant 0$ controla a influência de energia da área. Ao integrarmos $E(\omega)$ sobre $\mathcal{S}$ obteremos a distorção total, combinando área e ângulo, causada pela parametrização $\phi$.

O cálculo da energia $E$ no modo discreto, realizado numa superfície triangularizada que é uma superfície linear por partes, fica restrito a cada mapeamento entre os triângulos desta superfície tridimensional e da triangulação planar associada fazendo com que $E$ seja constante dentro de cada triângulo.

Ao considerarmos $T_{\mathcal{P}}$ e $T_{\mathcal{S}}$ o conjunto dos triângulos, respectivamente, da triangulação planar $\mathcal{P}$ e da superfície tridimensional $\mathcal{S}, T_{\mathcal{P}}(t)$ um triângulo de $T_{\mathcal{P}}$ e $T_{\mathcal{S}}(t)$ um triângulo de $T_{\mathcal{S}}$ podemos definir a parametrização restrita ao triângulo $T_{\mathcal{P}}(t)$ como $\left.\phi\right|_{T_{\mathcal{P}}(t)}: T_{\mathcal{P}}(t) \rightarrow T_{\mathcal{S}}(t)$.

Como apresentado em [17] o cálculo da energia discreta do ângulo restrita à aplicação $\left.\phi\right|_{T_{\mathcal{P}}(t)}$ é dado por

$$
E_{\text {ângulo }}=\frac{\cot \alpha|a|^{2}+\cot \beta|b|^{2}+\cot \gamma|c|^{2}}{2 \text { área }\left(T_{\mathcal{P}}(t)\right)}
$$

onde $a, b$ e $c$ são as medidas dos lados do triângulo $T_{\mathcal{P}}(t)$ e $\alpha, \beta$ e $\gamma$ são os ângulos internos do triângulo $T_{\mathcal{S}}(t)$.

Adicionalmente, em [11] é introduzida a energia discreta da área restrita à aplicação $\left.\phi\right|_{T_{\mathcal{P}}(t)}$ dada por

$$
E_{\text {área }}=\frac{\text { área }\left(T_{\mathcal{S}}(t)\right)}{\text { área }\left(T_{\mathcal{P}}(t)\right)}+\frac{\text { área }\left(T_{\mathcal{P}}(t)\right)}{\text { área }\left(T_{\mathcal{S}}(t)\right)}
$$

e ainda a energia total discreta da distorção causada por $\phi$ combinando as energias da área e do ângulo dada por

$$
E(\phi)=\sum E_{\text {ângulo }} \cdot\left(E_{\text {área }}\right)^{\theta} \text {.área }\left(T_{\mathcal{S}}(t)\right)
$$

onde para cada parcela da soma as energias são calculadas, respectivamente, com as coordenadas do triângulo $T_{\mathcal{S}}(t)$ e do triângulo associado biunivocamente $T_{\mathcal{P}}(t)$ e $\theta \geqslant 0$.

\section{OTIMIZAÇÃo HIERÁRQUICA}

Inicialmente consideramos a estrutura hierárquica de uma superfície triangularizada $\mathcal{S}$ e partimos do nível $H_{0}$ que é associado pela parametrização com a triangulação planar $\mathcal{P}_{0}$, resultante da triangulação $\mathcal{P}$ após o processo de simplificação.

Neste nível $H_{0}$ calculamos a energia de cada vértice que equivale à energia total restrita à estrela do vértice, ou seja, a soma indicada na equação $(*)$ é realizada apenas nos triângulos da estrela deste vértice. Em cada iteração os vértices são ordenados de acordo com a sua energia de modo que o vértice de maior energia fica com a prioridade. Além disso, nesta iteração otimizamos o funcional $E(\phi)$ em relação às coordenadas locais de cada vértice selecionado seguindo a ordenação pelas energias e tendo como restrição a vizinhança fixada desse vértice composta pelas coordenadas locais dos vértices pertencentes ao link de sua estrela. Os vértices pertencentes a este link são bloqueados e não serão selecionados na mesma iteração para a otimização. Desse modo, em cada iteração a posição geométrica correspondente na triangulação planar de cada vértice selecionado será alterada e a energia destes vértices serão reduzidas.

Avançamos progressivamente com a hierarquia até atingir o nível máximo e para cada nível selecionado são realizadas $m$ iterações deste processo de otimização. Em cada iteração é realizada a ordenação dos vértices de acordo com as energias, a otimização da energia de cada vértice não bloqueado e os respectivos bloqueios dos vértices pertencentes a vizinhança de cada vértice selecionado para otimização.

No exemplo ilustrado pela figura 4 utilizamos o modelo Júlio César que foi preparado para ter a topologia de um disco, composto por 13721 vértices e 27136 faces. Após a simplificação e construção da hierarquia o nível $H_{0}$ ficou composto por 974 vértices e 1642 faces e aplicamos o processo 
de otimização hierárquica nos níveis $H_{0}, H_{3186}, H_{6373}, H_{9560}$ e na superfície original $\mathcal{S}_{12747}$ usando $\theta=1.0$ no funcional de otimização. O processo de simplificação e construção da hierarquia foi realizado em 0.44 segundos.

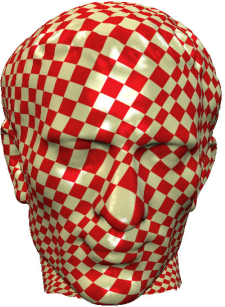

(a)

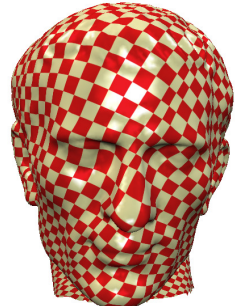

(b)

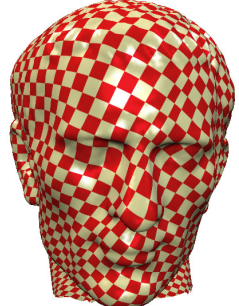

(c)

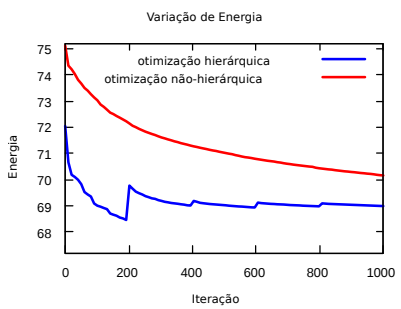

(d)

Figura 4. (a) textura no modelo original, (b) textura com coordenadas otimizadas sem hierarquia, (c) textura com coordenadas otimizadas pela hierarquia e (d) variação de energias

O gráfico 4d compara a redução da energia total de deformação obtida pela otimização apenas na superfície original, curva em vermelho e, em seguida, pelo processo de otimização hierárquica, curva em azul. Neste gráfico, o eixo horizontal marca o número de iterações e o eixo vertical marca a soma das energias de todos os vértices. Na superfície original foram realizadas 1000 iterações do processo de otimização e o tempo de processamento foi 6.46 minutos, já na hierarquia foram realizadas cinco etapas com 200 iterações nos quatro níveis selecionados e, por fim, na superfície original. O tempo de processamento pela hierarquia foi 3.38 minutos. A redução de energia apresentada pelo gráfico pode ser observada comparando a figura $4 \mathrm{c}$ com a figura $4 \mathrm{~b}$, esta última foi obtida com a otimização não-hierárquica. $\mathrm{O}$ processador utilizado nesta aplicação foi o Intel Core i7-7500U e $2.70 \mathrm{GHz}$ x4 com $8 \mathrm{~GB}$ de memória RAM.

\section{CONCLUSÃO}

Este artigo apresentou uma parte do que já temos estudado sobre a parametrização hierárquica de superfícies triangularizadas topologicamente equivalentes ao disco. Nosso objetivo na pesquisa está prosseguindo para a construção de uma estrutura hierárquica mais geral, que englobe superfícies compactas sem bordo e que reduza melhor as distorções introduzidas.

Apresentamos uma estrutura hierárquica que modifica a hierarquia apresentada por [8] e permite uma liberdade maior para escolher vértices a serem colapsados na simplificação da superfície, evitando possíveis inconsistências topológicas ou de orientação das faces. Nesse sentido, ainda buscaremos comparar melhor os nossos resultados obtidos.

Com o objetivo de reduzir as distorções com mais eficiência, aplicamos um processo de otimização em nossa estrutura hierárquica e já obtivemos resultados que mostram a melhoria em tempo de processamento da otimização e a redução da energia de distorção se comparado com o processo de otimização realizado na triangulação original sem a estrutura hierárquica. Desejamos ainda comparar com mais precisão o comportamento do processo de otimização aplicado em nossa estrutura hierárquica e aplicado na hierarquia proposta por [8].

A independência para a escolha dos vértices a serem otimizados nos permite modificar o processamento serial para o processamento da otimização em paralelo. Esta é outra modificação importante que estamos buscando implantar observando o ganho em eficiência computacional.

\section{REFERÊNCIAS}

[1] M. Siqueira, D. Xu, J. Gallier, L. G. Nonato, D. M. Morera, and L. Velho, "A new construction of smooth surfaces from triangle meshes using parametric pseudo-manifolds," Computers \& Graphics, vol. 33, no. 3, pp. 331-340, 2009.

[2] E. de Medeiros Filho and M. Siqueira, "Good random multi-triangulation of surfaces," IEEE transactions on visualization and computer graphics, 2017.

[3] M. S. Floater and K. Hormann, "Surface parameterization: a tutorial and survey," in Advances in multiresolution for geometric modelling. Springer, 2005, pp. 157-186.

[4] K. Hormann, B. Lévy, and A. Sheffer, "Mesh parameterization: Theory and practice," 2007.

[5] A. Sheffer, E. Praun, and K. Rose, "Mesh parameterization methods and their applications," Foundations and Trends $\AA$ in Computer Graphics and Vision, vol. 2, no. 2, pp. 105-171, 2007. [Online]. Available: http://dx.doi.org/10.1561/0600000011

[6] F. Payan, C. Roudet, and B. Sauvage, "Semi-regular triangle remeshing: A comprehensive study," in Computer Graphics Forum, vol. 34, no. 1. Wiley Online Library, 2015, pp. 86-102.

[7] H. Hoppe, "Progressive meshes," in Proceedings of the 23rd Annual Conference on Computer Graphics and Interactive Techniques, ser. SIGGRAPH '96. New York, NY, USA: ACM, 1996, pp. 99-108. [Online]. Available: http://doi.acm.org/10.1145/237170.237216

[8] K. Hormann, G. Greiner, and S. Campagna, "Hierarchical parametrization of triangulated surfaces," in Proceedings of Vision, Modeling, and Visualization, vol. 1999, 1999, pp. 219-226.

[9] M. Botsch, L. Kobbelt, M. Pauly, P. Alliez, and B. Lévy, Polygon mesh processing. AK Peters/CRC Press, 2010.

[10] M. P. Do Carmo, Differential Geometry of Curves and Surfaces: Revised and Updated Second Edition. Courier Dover Publications, 2016.

[11] P. Degener, J. Meseth, and R. Klein, "An adaptable surface parameterization method." IMR, vol. 3, pp. 201-213, 2003.

[12] W. T. Tutte, "How to draw a graph," Proceedings of the London Mathematical Society, vol. 3, no. 1, pp. 743-767, 1963.

[13] M. S. Floater, "Parametrization and smooth approximation of surface triangulations," Computer aided geometric design, vol. 14, no. 3, pp. 231-250, 1997.

[14] M. Floater, "One-to-one piecewise linear mappings over triangulations," Mathematics of Computation, vol. 72, no. 242, pp. 685-696, 2003.

[15] M. S. Floater, "Mean value coordinates," Computer aided geometric design, vol. 20, no. 1, pp. 19-27, 2003.

[16] O. Devillers, "On deletion in delaunay triangulations," International Journal of Computational Geometry \& Applications, vol. 12, no. 03 , pp. 193-205, 2002.

[17] K. Hormann and G. Greiner, "Mips: An efficient global parametrization method," France on 1-7 July 1999. Proceedings, Volume 1. Curve and Surface Design. F61775-99-WF068, p. 153, 2000. 